\title{
Role of dexmedetomidine infusion after coronary artery bypass grafting
}

\author{
Tamer Hamouda ${ }^{1,2}$, Mohamed Ismail ${ }^{1,3}$, Tamer Hamed Ibrahim ${ }^{4}$, Hesham Ewila ${ }^{5}$ and Ahmed Elmahrouk ${ }^{1,6^{*}}$ (D)
}

\begin{abstract}
Background: Postoperative pain has negative consequences on patients' outcomes after cardiac surgery. Routine management with opioid and or non-steroidal anti-inflammatory medications has several disadvantages. Dexmedetomidine is a selective a2 agonist used for sedation and analgesia. The use of dexmedetomidine for postoperative pain management and decreasing delirium and agitation in cardiac surgery patients is a matter of debate. Our objective was to determine the role of an early administration of dexmedetomidine in decreasing opioid use post-cardiac surgery and its effects on the quality of postoperative recovery.

Results: Medical records of 120 patients admitted to the cardiac surgery intensive care unit (CSICU) after coronary artery bypass grafting (CABG) in two cardiac centers between December 2015 and December 2016 were reviewed. Patients were divided into two groups. Group A included 55 patients who received dexmedetomidine in a dose of $0.2-0.4 \mathrm{mcg} / \mathrm{kg} / \mathrm{h}$ on admission to CSICU, and group B included 65 patients who did not receive dexmedetomidine. The primary outcome was the pain score immediately after extubation, and the secondary outcomes included post-extubation sedation and pain scores for $12 \mathrm{~h}$.

There were significant decrease of the pain scores in dexmedetomidine group that continues through the 3rd, 6 th, 8th, and 12th hour readings after surgery with mean modified Ramsay scores $0.1 \pm 0.0,0.89 \pm 2.05,0.35 \pm 0.1$, and $0.12 \pm 1.1$ respectively compared to $0.46 \pm 1.15,3.46 \pm 2.93,0.98 \pm 1.90$, and $0.12 \pm 1.1$ in group $B(p<0.001)$, significant decrease in cumulative morphine received $(p<0.001, \mathrm{OR}=909,95 \% \mathrm{Cl} 0.05-0.19)$, favorable reduction in heart rate in dexmedetomidine group $(80 \pm 1.9 \mathrm{~b} / \mathrm{min})$ compared to $96 \pm 8.8 \mathrm{~b} / \mathrm{min}$ in the other group ( $p=0.017)$, and smoother recovery from general anesthesia.

Conclusion: Administration of dexmedetomidine in the early postoperative period can be safe. It may reduce the use of opioids, has sedative, analgesic, and sympatholytic effects that could play a useful role during the management of coronary artery bypass patients, and may improve postoperative recovery.
\end{abstract}

Keywords: Dexmedetomidine, Coronary artery bypass grafting, Postoperative pain, Alpha 2 agonists

\section{Background}

Postoperative pain after cardiac surgery has a negative impact on the outcome, in addition to the patients' experience. Tachycardia, hypertension, arrhythmias, and myocardial ischemia are frequent postoperative findings. Most postoperative pain management protocols are opioid-based, which have a dose-dependent analgesic effect [1].

\footnotetext{
* Correspondence: ahmed.elmahrouki1@med.tanta.edu.eg; Ael-

mahrouk@kfshrc.edu.sa; a_marouky@hotmail.com

${ }^{1}$ Cardiovascular Department, King Faisal Specialist Hospital and Research

Center, Jeddah, Saudi Arabia

${ }^{6}$ Cardiothoracic Surgery Department, Faculty of Medicine, Tanta University,

Tanta, Egypt

Full list of author information is available at the end of the article
}

On the other hand, opioids have a number of undesired side effects, including nausea, vomiting, decreased gastrointestinal motility, respiratory depression, drowsiness, and hemodynamic effect with large doses [2]. Moreover, nonopioids analgesics, such as non-steroidal anti-inflammatory drugs and acetaminophen, may be a useful complement to opioids for postoperative pain relief. Additionally, nonopioid analgesics can reduce the need for opioids and minimize its side effects. However, the efficiency of these medications can be limited, as it can seriously affect the patients postoperatively because of their effect on bleeding and renal function [3].

Dexmedetomidine (DEX) is a highly selective $\alpha 2$ receptor agonist that provides better sedation, analgesic, and 
anxiolytic effect. This property is considered unique among sedatives used for intensive care units generally [4, 5]. Many studies on DEX in postoperative high-risk noncardiac patients showed that those patients required significantly lower sedative agents. Furthermore, dexmedetomidine has a less hemodynamic effect and non-significant respiratory depression [6,7]. Additionally, patients who received DEX experienced mutual sedation and smooth extubation, and they had lower mortality [7].

Moreover, recent findings show that such patients have a lower incidence of delirium over comparators [8, 9]. On the other hand, other studies argued the effect of DEX in decreasing the incidence of postoperative delirium, especially in older patients $[10,11]$. In this retrospective study, we tried to examine the efficacy of postcoronary artery bypass grafting (CABG) DEX infusion in reducing postoperative morphine consumption and in improving the quality of postoperative recovery.

\section{Methods}

\section{Design and patients}

Data of all patients admitted to the cardiac surgery intensive care unit (CSICU) following CABG, between December 2015 and 2016, were retrieved from the medical records after approval from the local medical ethics committee. Patients' confidentiality was maintained by removing any identifying information from the data set before further usage and analysis. The variables and their values were coded into an alphanumeric format for concealment with few designated persons having the coding key.

A hundred and twenty patients were included in the study. Patients with renal failure, prolonged postoperative intubation for more than $8 \mathrm{~h}$ because of hemodynamic instability or bleeding, and patients who had re-exploration were excluded.

Patients were divided into two groups: group A included 54 patients who received postoperative DEX infusion for sedation, and group B included 66 patients who did not receive DEX.

\section{Study protocol and outcomes}

All patients were intubated, ventilated, and sedated by propofol intravenous (IV) infusion $25-50 \mu \mathrm{g} / \mathrm{kg} / \mathrm{min}$. On admission to CSICU, vital signs were recorded, and group A patients were started on DEX IV infusion 0.2$0.4 \mu \mathrm{g} / \mathrm{kg} / \mathrm{h}$. while patients in group B were maintained only on propofol infusion.

Vital signs, including heart rate (HR), oxygen saturation, and end-tidal carbon dioxide, were continuously monitored, and blood pressure was monitored both invasively and non-invasively. Patients were started on acetaminophen $1 \mathrm{~g}$ IV every $6 \mathrm{~h}$, and $2-3 \mathrm{mg}$ of morphine were administered intravenously if the pain was suspected in case of unexplained tachycardia and hypertension when the patients were still intubated.

We reviewed the difference in total morphine consumption and vital data between the two groups while patients were intubated. Our protocol was to start weaning the patients from sedation and prepare for extubation $2 \mathrm{~h}$ after admission to CSICU and after ensuring the stable hemodynamic and full return of muscle power, so once these criteria were met, propofol and DEX infusion were turned off, and patients were extubated after full recovery.

Our primary outcome was the pain score assessed by the primary nurse and physician immediately after extubation. The pain was evaluated according to the numeric pain intensity scale (NPIS), ranging from no pain (0) to severe non-tolerable pain (10).

We reviewed the pain scores and vital signs every hour in all patients for the first $12 \mathrm{~h}$ post-extubation in addition to sedation score which was assessed by modified Ramsay score (MRS) and ranged from 1 to 6 as follows: 1 = anxious/agitated, 2 = cooperative oriented, $3=$ responds to commands only, $4=$ asleep with brisk response to light glabellar tap (LGT), $5=$ sluggish response to (LGT), 6 = asleep no response to (LGT).

Our protocol was to administer $2-3 \mathrm{mg}$ of morphine to the patient once the pain score is $\geq 2$, and the total morphine consumption in the first $12 \mathrm{~h}$ post-extubation in both groups was reviewed, and that was our secondary outcome in addition to the vital data and sedation scores in the first $12 \mathrm{~h}$ after extubation.

\section{Statistical analysis}

Statistical analysis was performed using SPSS 15 for Windows (IBM Corp, Chicago, IL, USA). Continuous data were presented as mean \pm standard deviation and categorical data as count and percent. $T$ test was used to compare the continuous variables, and chi-squared test or fisher exact test was used for categorical variables when appropriate. The trend of pain severity was investigated by a repeated measure analysis of variance (ANOVA) model. Freedom from the first dose of morphine was plotted using the Kaplan-Meier survival curve and compared between both groups using a log-rank test.

\section{Results}

One hundred and twenty patients with a mean age of $56 \pm 10.1$ years were included in the study. The mean left ventricular ejection fraction (LVEF) was $40 \pm 9.4 \%$ in the DEX group compared to $44 \pm 7.3 \%$ in group B $(p=0.75)$. The average time of anesthesia was $312 \pm 66 \mathrm{~min}$ in group (A) and $309 \pm 42 \mathrm{~min}$ in group (B) $(p=0.54)$. Patients stayed in the intensive care unit (ICU) after surgery with a mean time of $2.9 \pm 1.1$ days and a range of 2 to 4 days. 
Fifty-four patients had intravenous DEX started on admission to the intensive care unit, and 66 patients were started on propofol. Analysis of baseline variables of the patients was presented in Table 1, which showed no significant differences between the two groups.

The severity of pain was measured by 10 points in NPIS in different time intervals: 1st, 3rd, 6th, 12th, 18th, and 24th hours (Table 2).

Patients experienced a period of analgesia after surgery. The most severe pain had a point that was less than 3. By the 6th hour after surgery, the pain started to appear gradually and became more severe. The severity of pain diminished until the disappearance at the 18th hour after surgery $(p<0.001)$.

In the first hour after the operation, the trend of pain severity was equal in both groups. The effect of analgesia continues in those who received dexmedetomidine until the 3rd hour after surgery, while in the control group, the pain severity was increasing. The patients in the DEX group had statistically significant lower pain severity compared to the control group throughout the first postoperative day ( $p$ value $<0.001$ ) (Fig. 1).

The assessment was done every hour during intubation and 2-hourly after extubation; the mean sedation score was analyzed all through intubation (Table 3 ). We observed that intubation time was $6 \pm 2.3 \mathrm{~h}$ in the DEX group compared to group B with $8 \pm 7.9 \mathrm{~h}(p=0.231)$. The mean arterial pressure had increased in both groups within the first $1 \mathrm{~h}$ and dropped within the first $2 \mathrm{~h}$ then became stable in the DEX group with a lower heart rate than the other group.

In the DEX group, $10 \%$ of patients needed to receive morphine for pain compared to $95 \%$ of the patients in the other group $(p<0.001, \mathrm{OR}=909,95 \%$ CI $0.05-0.19)$.
Moreover, the dose of received morphine sulfate was lower in the DEX group $(1.0 \pm 1.2 \mathrm{mg})$ compared to $2.9 \pm 1.8 \mathrm{mg}$ in the other group $(p<0.001)$.

The time of prescription of the 1st dose of morphine sulfate could be considered as a proxy for the beginning of intolerable pain (pain score between 4 and 5) (Fig. 2). The pain became relatively unbearable $6 \mathrm{~h}$ after finishing the surgery. No significant difference between the two groups was noticed using the log-rank test $(p=0.09)$. The pattern of receiving the first dose of morphine sulfate was similar in both groups.

\section{Discussions}

This study demonstrated that early infusion of DEX following CABG operations was associated with a reduction of morphine usage, a decrease in pain severity, and improvement of sedation, which encouraged early extubation. Pain control is crucial after cardiac surgery. The release of catecholamine causes the increase of the peripheral vascular resistance, heart rate, and risk of myocardial infarction, arrhythmias, and mortality. From our observations, we noticed a transient rise in main arterial pressure (MAP) during the 1st postoperative hour in both groups, followed by a decrease in MAP.

Arain and associates [12] found a brief increase in blood pressure in the DEX group, which was related to activation of $\alpha 2$ agonist on the smooth muscle of the vessel wall, which leads to transient vasoconstriction and increased in mean arterial pressure. We have noticed this in both groups. However, we thought these initial hemodynamic changes in MAP and HR were most probably related to post-surgical stress, the surgical procedures itself, or pre-existing hypertension as it happened in both groups [13]. The decrease in MAP and HR was

Table 1 Comparison of the preoperative, operative, and postoperative data between groups (continuous data are presented as mean and standard deviation or median and categorical data as number and percent)

\begin{tabular}{llll}
\hline & Group A ( $n=54)$ & Group B $(n=66)$ & $p$ value \\
\hline Age (years) & $54 \pm 9.5$ & $57 \pm 8.3$ & 0.181 \\
Weight (kg) & $85 \pm 10$ & $80 \pm 9.3$ & 0.530 \\
Height (cm) & $1.61 \pm 9$ & $165 \pm 3$ & 1.511 \\
Ejection fraction & $40 \pm 9.4$ & $44 \pm 7.3$ & 0.752 \\
Duration of anesthesia (min) & $312 \pm 66$ & $309 \pm 42$ & 0.541 \\
Operative time (min) & $248 \pm 18$ & $251 \pm 92$ & 0.572 \\
Bypass time (min) & $110 \pm 38.5$ & $105 \pm 40.3$ & 0.641 \\
ICU stay/days & $2 \pm 0.5$ & $3 \pm 0.1$ & 0.512 \\
Intubation time (hours) & $6 \pm 2.3$ & $8 \pm 7.9$ & 0.231 \\
MAP (mmHg) & $77.93 \pm 5.9$ & $74.53 \pm 8.3$ & 0.561 \\
Heart rate & $80 \pm 1.9$ & $96 \pm 8.8$ & 0.017 \\
Modified RS (median) & 2 & 3 & 0.802 \\
Pain intensity score median & 1 & 2 & 0.113 \\
\hline Cm centimeters, min minutes, ICU intensive care unit, MAP mean arterial pressure HR heart rate, Modified RS modified Ramsa sCore
\end{tabular}

$\mathrm{cm}$ centimeters, min minutes, ICU intensive care unit, MAP mean arterial pressure, $H R$ heart rate, Modified RS modified Ramsay score 
Table 2 Pain score in different time interval after cardiac surgery (data are presented as mean and standard deviation)

\begin{tabular}{|c|c|c|c|c|c|c|}
\hline Group & 1st hour & 3rd hour & 6th hour & 8th hour & 12th hour & 24th hour \\
\hline Group A $(n=54)$ & $0.39 \pm 1.25$ & $0.1 \pm 0.0$ & $0.89 \pm 2.05$ & $0.35 \pm 0.1$ & $0.12 \pm 1.1$ & $0.0 \pm 0.90$ \\
\hline Group B $(n=66)$ & $1.08 \pm 0.67$ & $0.46 \pm 1.15$ & $3.46 \pm 2.93$ & $0.98 \pm 1.90$ & $0.30 \pm 0.2$ & $0.0 \pm 0.96$ \\
\hline
\end{tabular}

related to more gradual central effect of DEX in patients who received it, while the reduction of MAP for patients who did not receive DEX and depended on morphine as main analgesic most probably was related to decreasing catecholamine and direct vasodilatation effect of morphine considering that there was no significant difference in the number of patients receiving inotropes and vasodilators between the two groups.

Our findings were in line with Liu and coworkers' [14] meta-analysis, which stated that DEX lead to a shorter length of intubation, but it can be associated with bradycardia in patients after cardiac surgery compared with propofol. This tendency to lower the heart rate and systolic blood pressure with decreased incidence of tachycardia and arrhythmias have a cardioprotective effect, as stated by another meta-analysis by Gong and coauthors [15].

On the other hand, Mukhtar and colleagues [16] concluded in their study that intraoperative DEX infusion attenuated the hemodynamic and neuroendocrine response to surgical trauma and cardiopulmonary bypass. The same findings were declared by Priye and associates [17], who concluded that DEX infusion, even those without the loading dose, have a safe, effective adjuvant analgesic effect. It can reduce narcotic consumption without undesirable hemodynamic effects in cardiac surgery patients.

Shehabi and coworkers [11] found that DEX reduced vasopressors' requirement after cardiac surgery with effective analgesia sedation, less hypertension, and more bradycardia versus morphine regimen. In our study, we did not find any difference between the two groups with regard to MAP and HR ( $p=0.561$ and 0.017, respectively). Barletta and associates [18] stated that DEX decreased cardiac index and HR for patients undergoing lengthy procedures which remained reduced at the time of discharge; our results did not support this conclusion as there was no significant difference between the two groups in HR. On the other hand, Maldonado and coworkers [19] concluded that DEX was associated with a lower rate of respiratory depression. It caused a higher rate of adverse hemodynamic events, which might be a concern in a hemodynamically unstable patient, but we did not observe any adverse effect on hemodynamic stability; this can be attributed to the low number of patients who needed inotropic support or vasodilator during the post-operative period in our cohort. Moreover, we have estimated opioid administration as analgesics postoperatively, which could reflect passively on the patients' hemodynamics post-CABG. We observed that $10 \%$ of the patients were in need of opioids in the DEX group. These patients could be considered to have a low pain threshold or the post-operative managing doctors had a low threshold to give his patients more analgesia.

It worth mentioning that, while $95 \%$ of the control group were depended on opioids, $5 \%$ only of them only received non-steroidal anti-inflammatory (NSAID), according to the

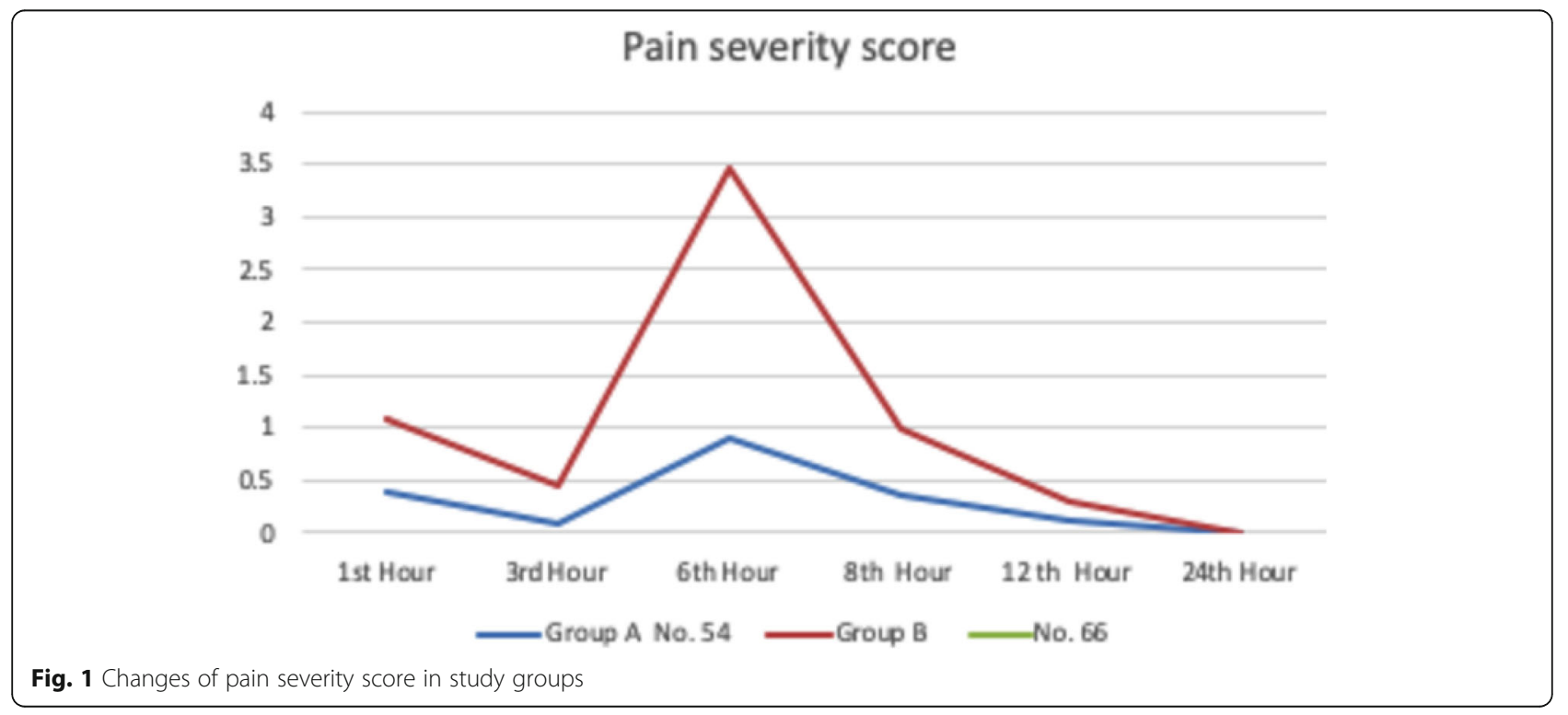


Table 3 Sedation score during intubation and after extubation (data are presented as the mean)

\begin{tabular}{|c|c|c|c|c|c|c|}
\hline & $\begin{array}{l}\text { During } \\
\text { intubation }\end{array}$ & $\begin{array}{l}\text { Immediately after } \\
\text { extubation }\end{array}$ & $\begin{array}{l}2 \mathrm{~h} \text { after } \\
\text { extubation }\end{array}$ & $\begin{array}{l}4 \mathrm{~h} \text { after } \\
\text { extubation }\end{array}$ & $\begin{array}{l}6 \mathrm{~h} \text { after } \\
\text { extubation }\end{array}$ & $\begin{array}{l}8 \mathrm{~h} \text { after } \\
\text { extubation }\end{array}$ \\
\hline $\begin{array}{l}\text { Group A }(n= \\
54)\end{array}$ & 5.2 & 3.2 & 2.9 & 2.7 & 2.2 & 2.3 \\
\hline $\begin{array}{l}\text { Group B }(n= \\
66)\end{array}$ & 4.5 & 2.1 & 2.1 & 2 & 2.1 & 2.1 \\
\hline
\end{tabular}

assessment of the post-operative ICU doctors who considered most of these patients with high pain threshold, and so, NSAID was enough to control their pain. Moreover, the dose of opioids given in the DEX group was significantly less than those of the other group [20, 21].

The pattern of pain in both groups was almost the same, which means that DEX does not alter the trend of the pain but decreased the intensity, and both groups needed to receive analgesics at the same point according to pain score between 4 and 5 . These results agreed with Cheng and associates' study [22].

Some authors mentioned that the use of DEX decreased the length of ICU stay in comparison with the use of other sedative and analgesics like midazolam and morphine, and we did not observe in our study significant differences in ICU stay as it was $2 \pm 0.5$ and $3 \pm 0.1$ days $(p=0.512)$. There are many factors other than pain and analgesics which could influence the ICU stay, including the patient's hemodynamics, which can be affected by other etiologies other than pain.
From the clinical point of view, Lin and coworkers [9] suggested that DEX gave a suitable condition through which it facilitated weaning process from mechanical ventilator as it did not depress spontaneous ventilation, as well as it decreased the risk of delirium, ventricular tachycardia, and hyperglycemia following cardiac surgery; however, it could cause significant bradycardia. In our study, there was no significant difference between the two groups in intubation time.

\section{Study limitations}

The primary limitation of the study is the retrospective nature, and several factors may have affected the outcomes other than the treatment. A randomized clinical trial is required to adjust for the measured and unmeasured variables that could confound the results. However, because the administration of DEX in our cohort was not related to patients' specific risk factors, the two groups had comparable baseline data.

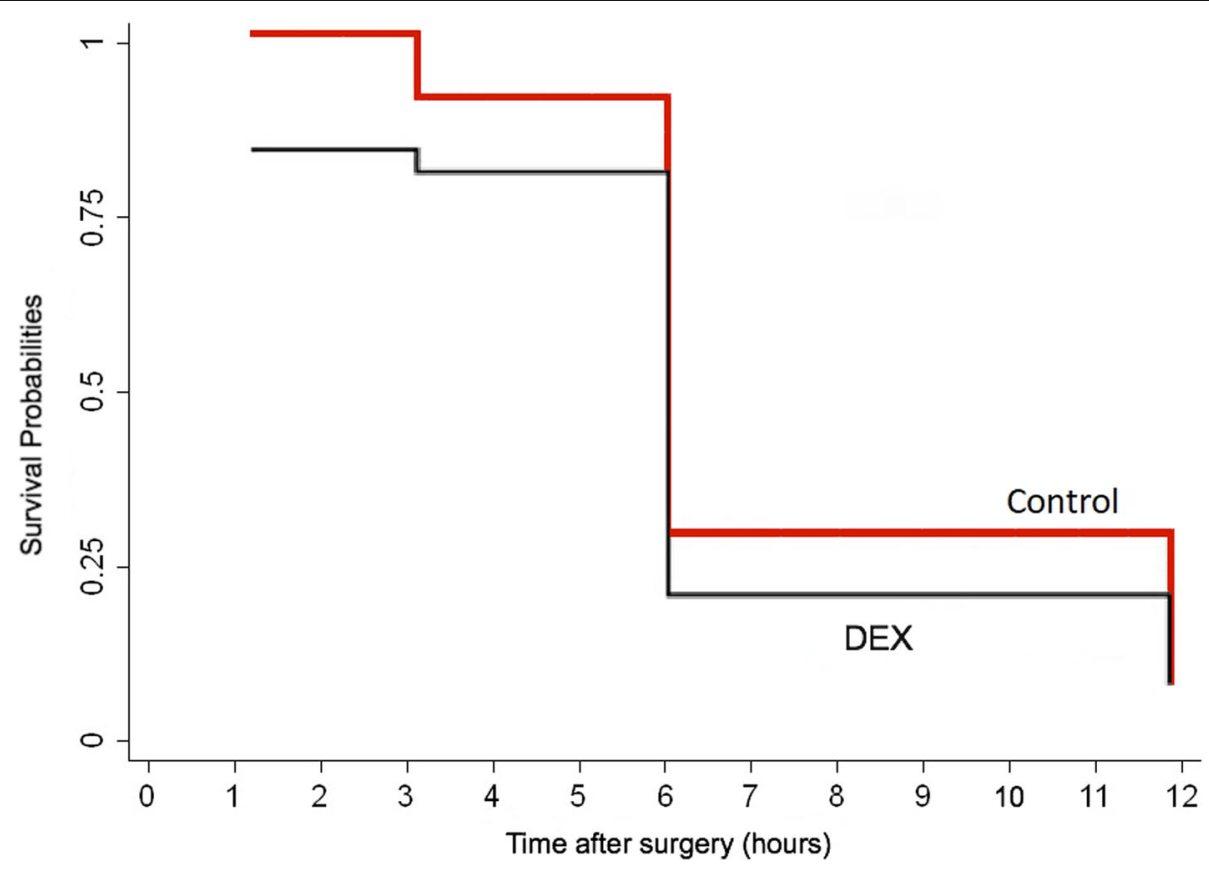

Fig. 2 Kaplan-Meier curve for estimation of the time to receive the morphine 


\section{Conclusion}

Administration of dexmedetomidine in the early postoperative period can be safe. It may reduce the use of opioids, has sedative, analgesics, and sympatholytic effects that could play a useful role during the management of coronary artery bypass patients, and may improve postoperative recovery.

\section{Abbreviations}

CABG: Coronary artery bypass grafting; CSICU: Cardiac surgery intensive care unit; DEX: Dexmedetomidine; HR: Heart rate; ICU: Intensive care unit; LGT: Light glabellar tap; LVEF: Left ventricular ejection fraction; MAP: Main arterial pressure; MRS: Modified Ramsay score; NPIS: The numeric pain intensity scale; NSAID: Non-steroidal anti-inflammatory

\section{Acknowledgements}

Not applicable.

\section{Authors' contributions}

$\mathrm{TH}$ conducted the literature search and data collection and drafted the manuscript. Ml designed the study, analysis, and interpretation of data and drafted the manuscript. TI conducted the statistical analysis and interpretation of data and drafted the manuscript. HE conducted the statistical analysis and data collection and conducted the literature search and interpretation of data. AE supervised the study and conducted the review of data, was involved in the study design, analysis, and interpretation of data, and drafted the manuscript (corresponding author). All authors have read and approved the manuscript.

\section{Funding}

This research received no specific grant from any funding agency in the public, commercial, or not-for-profit sectors.

\section{Availability of data and materials}

Data are available with the corresponding author upon request.

\section{Ethics approval and consent to participate}

The study was approved by the local institutional review board (IRB), at Alhada Military Hospital \# 2014, and the patients' consent was waived for this retrospective observational study.

\section{Consent for publication}

Not applicable.

\section{Competing interests}

The authors declare that they have no competing interests.

\section{Author details \\ ${ }^{1}$ Cardiovascular Department, King Faisal Specialist Hospital and Research Center, Jeddah, Saudi Arabia. ${ }^{2}$ Cardiothoracic Surgery Department, Faculty of Medicine, Benha University, Benha, Egypt. ${ }^{3}$ Cardiothoracic Surgery Department, Faculty of Medicine, Mansoura University, Mansoura, Egypt. ${ }^{4}$ Department of Anaesthesia, Faculty of Medicine, Ain Shams University, Cairo, Egypt. ${ }^{5}$ Department of Anaesthesia, Faculty of Medicine, Suez Canal University, Ismailia, Egypt. ${ }^{6}$ Cardiothoracic Surgery Department, Faculty of Medicine, Tanta University, Tanta, Egypt.}

Received: 5 December 2019 Accepted: 29 December 2019

Published online: 22 January 2020

\section{References}

1. Wijeysundera DN, Naik JS, Beattie WS (2003) Alpha-2 adrenergic agonists to prevent perioperative cardiovascular complications: a meta- analysis. Am J Med 114(9):742-752

2. Biccard BM, Goga S, de Beurs J (2008) Dexmedetomidine and cardiac protection for non-cardiac surgery: a meta-analysis of randomised control trials. Anaesthesia 63(1):4-14
3. Hoy SM, Keating GM (2011) Dexmedetomidine: a review of its use for sedation in mechanically ventilated patients in an intensive care setting and for procedural sedation. Drugs. 71:1481-1501

4. Christensen A (2009) Update on dexmedetomidine for adult ICU sedation. Conn Med 73:469-472

5. Szumita PM, Baroletti SA, Anger KE, Wechsler ME (2007) Sedation and analgesia in the intensive care unit: evaluating the role of dexmedetomidine. Am J Health Syst Pharm 64:37-44

6. Afonso J, Reis F (2012) Dexmedetomidine: current role on anaesthesia and intensive care. Rev Bras Anestesiol 62:118-133

7. Muntazar M, Kumar FC (2004) Cardiac arrest, a preventable yet a possible risk of dexmedetomidine: fact or fiction? Anesthesiology 101(6):1478-1479

8. Abd Aziz N, Chue MC, Yong CY, Hassan Y, Awaisu A, Hassan J, Kamarulzaman MH (2011) Efficacy and safety of dexmedetomidine versus morphine in post-operative cardiac surgery patients. Int J Clin Pharm 33: 150-154

9. Lin YY, He B, Chen J, Wang Z (2012) Can dexmedetomidine be a safe and efficacious sedative agent in post cardiac surgery patients? Critical car 16 R:169

10. Li X, Yang J, Nie XL, Zhang Y, Li XY, Li LH, Ma D (2017) Impact of dexmedetomidine on the incidence of delirium in elderly patients after cardiac surgery: a randomized controlled trial. PLoS One 12(2):e0170757

11. Shehabi $Y$, Grant $P$, Wolfenden $H$, Hammond N, Bass F, Campbell M, Chen J (2009) Prevalence of delirium with dexmedetomidine compared with morphine-based therapy after cardiac surgery: a randomized controlled trial (DEXmedetomidine COmpared to Morphine-DEXCOM Study). Anesthesiology 111(5):1075-1084

12. Arain SR, Ruehlow RM, Uhrich TD, Ebert TJ (2004) The efficacy of dexmedetomidine versus morphine for postoperative analgesia after major inpatient surgery. Anesth Analg 98(1):153-158

13. Ismail M, El-Mahrouk A, Hamouda T, Radwan H, Haneef A, Jamjoom A (2017) Factors influencing postoperative atrial fibrillation in patients undergoing on-pump coronary artery bypass grafting, single center experience. J Cardiothorac Surg 12:40

14. Liu X, Xie G, Zhang K, Song S, Song F, Jin Y, Fang X (2017) Dexmedetomidine vs propofol sedation reduces delirium in patients after cardiac surgery: a meta-analysis with trial sequential analysis of randomized controlled trials. J Crit Care 38:190-196

15. Gong Z, Ma L, Zhong YL, Li J, Lv J, Xie YB (2017) Myocardial protective effects of dexmedetomidine in patients undergoing cardiac surgery: a meta-analysis and systematic review. Exp Ther Med 13(5):2355-2361

16. Mukhtar AM, Obayah EM, Hassona AM (2006) The use of dexmedetomidine in paediatric cardiac surgery. Anaesthesia and Analgesia 103:52-56

17. Priye S, Jagannath S, Singh D, Shivaprakash S, Reddy DP (2015) Dexmedetomidine as an adjunct in postoperative analgesia following cardiac surgery: a randomized, double-blind study. Saudi J Anaesth 9(4): 353-358

18. Barletta JF, Miedema SL, Wiseman D, Heiser JC, KJ MA (2009) Impact of dexmedetomidine on analgesic requirements in patients after cardiac surgery in a fast-track recovery room setting. Pharmacotherapy 29:14271432

19. Maldonado J, van der Starre PJ and Wysong A: Postoperative sedation and the incidence of ICU delirium in cardiac surgery patients. Presented at the American Society of Anaesthesiologists, Annual Meeting. San Francisco, CA, Anesthesiology.2003; 99:A465

20. Elmahrouk A, Hamouda T, Kasab I, Ismail M, Jamjoom A (2017) Short term outcome of conventional versus off-pump coronary artery bypass grafting for high-risk patients. J Egypt Soc Cardiothorac Surg 26(1):24-29

21. Zientara A, Matter-Ensner S, Mariotti S, Seifert B, Graves K, Dzemali O, Genoni M (2016) Dexmedetomidine provides effective pain modulation and rapid extubation during perioperative fast-track management in patients after offpump coronary artery bypass grafting. Thorac Cardiovasc Surg 64:OP101

22. Cheng H, Li Z, Young N, Boyd D, Atkins Z, Ji F, Liu H (2016) The effect of dexmedetomidine on outcomes of cardiac surgery in elderly patients. J Cardiothorac Vasc Anesth 30(6):1502-1508

\section{Publisher's Note}

Springer Nature remains neutral with regard to jurisdictional claims in published maps and institutional affiliations. 